\title{
Reverses of Féjer's Inequalities for Convex Functions
}

\author{
SILVESTRU SEVER DRAGOMIR ${ }^{1,2}$
}

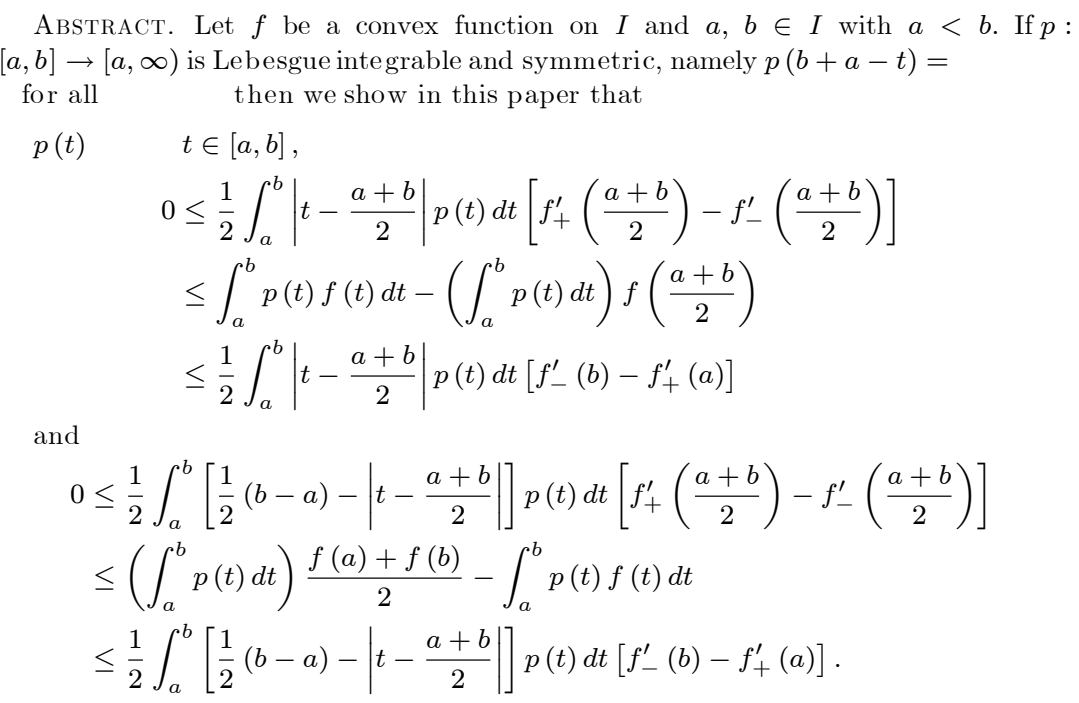

Keywords: convex functions; Integral inequalities; Hermite-Hadamard inequality; Féjer's inequalities

\section{Introduction}

The following inequality holds for any convex function $f$ defined on $\mathbb{R}$

$$
f\left(\frac{a+b}{2}\right) \leq \frac{1}{b-a} \int_{a}^{b} f(t) d t \leq \frac{f(a)+f(b)}{2}, \quad a, b \in \mathbb{R}, a<b .
$$

It was firstly discovered by Ch. Hermite in 1881 in the journal Mathesis (see [7]). But this result was nowhere mentioned in the mathematical literature and was not widely known as Hermite's result.

E. F. Beckenbach, a leading expert on the history and the theory of convex functions, wrote that this inequality was proven by J. Hadamard in 1893 [1]. In 1974, D. S. Mitrinović found Hermite's note in Mathesis [7]. Since (1.1) was known as Hadamard's inequality, the inequality is now commonly referred as the HermiteHadamard inequality. For a monograph devoted to this result see [5]. The recent survey paper [4] provides other related results.

Let $f:[a, b] \rightarrow \mathbb{R}$ be a convex function on $[a, b]$ and assume that $f_{+}^{\prime}(a)$ and $f_{-}^{\prime}(b)$ are finite. We recall the following improvement and reverse inequality for

1991 Mathematics Subject Classification. 26D15, 26D10.

Key words and phrases. Convex functions, Integral inequalities, Hermite-Hadamard inequality, Féjer's inequalities. 
the first Hermite-Hadamard result that has been established in [2]

$$
\begin{aligned}
0 & \leq \frac{1}{8}\left[f_{+}^{\prime}\left(\frac{a+b}{2}\right)-f_{-}^{\prime}\left(\frac{a+b}{2}\right)\right](b-a) \\
& \leq \frac{1}{b-a} \int_{a}^{b} f(t) d t-f\left(\frac{a+b}{2}\right) \leq \frac{1}{8}(b-a)\left[f_{-}^{\prime}(b)-f_{+}^{\prime}(a)\right] .
\end{aligned}
$$

The following inequality that provides a reverse and improvement of the second Hermite-Hadamard result has been obtained in [3]

$$
\begin{aligned}
0 & \leq \frac{1}{8}\left[f_{+}^{\prime}\left(\frac{a+b}{2}\right)-f_{-}^{\prime}\left(\frac{a+b}{2}\right)\right](b-a) \\
& \leq \frac{f(a)+f(b)}{2}-\frac{1}{b-a} \int_{a}^{b} f(t) d t \leq \frac{1}{8}(b-a)\left[f_{-}^{\prime}(b)-f_{+}^{\prime}(a)\right] .
\end{aligned}
$$

The constant $\frac{1}{8}$ is best possible in both (1.2) and (1.3).

In 1906, Fejér [6], while studying trigonometric polynomials, obtained inequalities which generalize that of Hermite \& Hadamard:

Theorem 1. Consider the integral $\int_{a}^{b} f(t) p(t) d t$, where $f$ is a convex function in the interval $(a, b)$ and $p$ is a positive function in the same interval such that

$$
p(a+t)=p(b-t), \quad 0 \leq t \leq \frac{1}{2}(b-a),
$$

i.e., $y=p(t)$ is a symmetric curve with respect to the straight line which contains the point $\left(\frac{1}{2}(a+b), 0\right)$ and is normal to the $t$-axis. Under those conditions the following inequalities are valid:

$$
f\left(\frac{a+b}{2}\right) \int_{a}^{b} p(t) d t \leq \int_{a}^{b} f(t) p(t) d t \leq \frac{f(a)+f(b)}{2} \int_{a}^{b} p(t) d t .
$$

If $f$ is concave on $(a, b)$, then the inequalities reverse in (1.4).

Clearly, for $p(t) \equiv 1$ on $[a, b]$ we get 1.1 .

If we take $p(t)=\left|t-\frac{a+b}{2}\right|, t \in[a, b]$ in Theorem 1 , then we have

$$
\frac{1}{4} f\left(\frac{a+b}{2}\right)(b-a)^{2} \leq \int_{a}^{b}\left|t-\frac{a+b}{2}\right| f(t) d t \leq \frac{f(a)+f(b)}{8}(b-a)^{2},
$$

for any convex function $f:[a, b] \rightarrow \mathbb{R}$.

We observe that, if we take $p(t)=(b-t)(t-a), t \in[a, b]$, then $p$ satisfies the conditions in Theorem 1, and by (1.4) we have the following inequality as well

$$
\frac{1}{6} f\left(\frac{a+b}{2}\right)(b-a)^{3} \leq \int_{a}^{b}(b-t)(t-a) f(t) d t \leq \frac{f(a)+f(b)}{12}(b-a)^{3},
$$

for any convex function $f:[a, b] \rightarrow \mathbb{R}$.

Motivated by the above results, in this paper we obtain an improvement and a reverse for each inequality in (1.4) and therefore generalize the Hermite-Hadamard inequalities (1.2) and (1.3). 


\section{Improvements and Reverse of FéJer Inequalities}

Following Roberts and Varberg [8, p. 5$]$, we recall that if $f: I \rightarrow \mathbb{R}$ is a convex function, then for any $x_{0} \in \stackrel{I}{I}$ (the interior of the interval $I$ ) the limits

$$
f_{-}^{\prime}\left(x_{0}\right):=\lim _{x \rightarrow x_{0}-} \frac{f(x)-f\left(x_{0}\right)}{x-x_{0}} \text { and } f_{+}^{\prime}\left(x_{0}\right):=\lim _{x \rightarrow x_{0}+} \frac{f(x)-f\left(x_{0}\right)}{x-x_{0}}
$$

exists and $f_{-}^{\prime}\left(x_{0}\right) \leq f_{+}^{\prime}\left(x_{0}\right)$. The functions $f_{-}^{\prime}$ and $f_{+}^{\prime}$ are monotonic nondecreasing on $\stackrel{\circ}{I}$ and this property can be extended to the whole interval $I$ (see [8, p. 7 ]).

From the monotonicity of the lateral derivatives $f_{-}^{\prime}$ and $f_{+}^{\prime}$ we also have the gradient inequality

$$
f_{-}^{\prime}(x)(x-y) \geq f(x)-f(y) \geq f_{+}^{\prime}(y)(x-y)
$$

for any $x, y \in \stackrel{\circ}{I}$.

If $I=[a, b]$, then at the end points we also have the inequalities

$$
f(x)-f(a) \geq f_{+}^{\prime}(a)(x-a)
$$

for any $x \in(a, b]$ and

$$
f(y)-f(b) \geq f_{-}^{\prime}(b)(y-b)
$$

for any $y \in[a, b)$.

We have the following refinement and reverse of Fejer's first inequality:

Theorem 2. Let $f$ be a convex function on $I$ and $a, b \in I$, with $a<b$. If $p$ : $[a, b] \rightarrow[a, \infty)$ is Lebesgue integrable and symmetric, namely $p(b+a-t)=p(t)$ for all $t \in[a, b]$, then

$$
\begin{aligned}
0 & \leq \frac{1}{2} \int_{a}^{b}\left|t-\frac{a+b}{2}\right| p(t) d t\left[f_{+}^{\prime}\left(\frac{a+b}{2}\right)-f_{-}^{\prime}\left(\frac{a+b}{2}\right)\right] \\
& \leq \int_{a}^{b} p(t) f(t) d t-\left(\int_{a}^{b} p(t) d t\right) f\left(\frac{a+b}{2}\right) \\
& \leq \frac{1}{2} \int_{a}^{b}\left|t-\frac{a+b}{2}\right| p(t) d t\left[f_{-}^{\prime}(b)-f_{+}^{\prime}(a)\right] .
\end{aligned}
$$

Proof. Let $a, b \in I$, with $a<b$. Using the integration by parts formula for Lebesgue integral, we have

$$
\begin{aligned}
& \int_{\frac{a+b}{2}}^{b}\left(\int_{t}^{b} p(s) d s\right) f^{\prime}(t) d t \\
& \left.=\left(\int_{t}^{b} p(s) d s\right) f(t)\right]_{\frac{a+b}{2}}^{b}+\int_{\frac{a+b}{2}}^{b} p(t) f(t) d t \\
& =-\left(\int_{\frac{a+b}{2}}^{b} p(s) d s\right) f\left(\frac{a+b}{2}\right)+\int_{\frac{a+b}{2}}^{b} p(t) f(t) d t
\end{aligned}
$$


and

$$
\begin{aligned}
& \int_{a}^{\frac{a+b}{2}}\left(\int_{a}^{t} p(s) d s\right) f^{\prime}(t) d t \\
& \left.=\left(\int_{a}^{t} p(s) d s\right) f(t)\right]_{a}^{\frac{a+b}{2}}-\int_{a}^{\frac{a+b}{2}} p(t) f(t) d t \\
& =\left(\int_{a}^{\frac{a+b}{2}} p(s) d s\right) f\left(\frac{a+b}{2}\right)-\int_{a}^{\frac{a+b}{2}} p(t) f(t) d t .
\end{aligned}
$$

By subtracting the second identity from the first, we get

$$
\begin{aligned}
& \int_{\frac{a+b}{2}}^{b}\left(\int_{t}^{b} p(s) d s\right) f^{\prime}(t) d t-\int_{a}^{\frac{a+b}{2}}\left(\int_{a}^{t} p(s) d s\right) f^{\prime}(t) d t \\
& =\int_{\frac{a+b}{2}}^{b} p(t) f(t) d t+\int_{a}^{\frac{a+b}{2}} p(t) f(t) d t \\
& -\left(\int_{\frac{a+b}{2}}^{b} p(s) d s\right) f\left(\frac{a+b}{2}\right)-\left(\int_{a}^{\frac{a+b}{2}} p(s) d s\right) f\left(\frac{a+b}{2}\right) .
\end{aligned}
$$

By the symmetry of $p$ we get

$$
\int_{\frac{a+b}{2}}^{b} p(s) d s=\int_{a}^{\frac{a+b}{2}} p(s) d s=\frac{1}{2} \int_{a}^{b} p(s) d s
$$

and then we can state the following identity of interest in itself

$$
\begin{aligned}
& \int_{a}^{b} p(t) f(t) d t-f\left(\frac{a+b}{2}\right) \int_{a}^{b} p(s) d s \\
& =\int_{\frac{a+b}{2}}^{b}\left(\int_{t}^{b} p(s) d s\right) f^{\prime}(t) d t-\int_{a}^{\frac{a+b}{2}}\left(\int_{a}^{t} p(s) d s\right) f^{\prime}(t) d t
\end{aligned}
$$

By the monotonicity of the derivative we have

$$
f_{+}^{\prime}(a) \leq f^{\prime}(t) \leq f_{-}^{\prime}\left(\frac{a+b}{2}\right), \text { for almost every } t \in\left(a, \frac{a+b}{2}\right)
$$

and

$$
f_{+}^{\prime}\left(\frac{a+b}{2}\right) \leq f^{\prime}(t) \leq f_{-}^{\prime}(b), \text { for almost every } t \in\left(\frac{a+b}{2}, b\right) .
$$

This implies

$$
\begin{aligned}
f_{+}^{\prime}(a)\left(\int_{a}^{t} p(s) d s\right) & \leq f^{\prime}(t)\left(\int_{a}^{t} p(s) d s\right) \\
& \leq f_{-}^{\prime}\left(\frac{a+b}{2}\right)\left(\int_{a}^{t} p(s) d s\right), t \in\left[a, \frac{a+b}{2}\right]
\end{aligned}
$$


and

$$
\begin{aligned}
f_{+}^{\prime}\left(\frac{a+b}{2}\right)\left(\int_{t}^{b} p(s) d s\right) & \leq f^{\prime}(t)\left(\int_{t}^{b} p(s) d s\right) \\
& \leq f_{-}^{\prime}(b)\left(\int_{t}^{b} p(s) d s\right), t \in\left[\frac{a+b}{2}, b\right]
\end{aligned}
$$

and by integration

$$
\begin{aligned}
f_{+}^{\prime}\left(\frac{a+b}{2}\right) \int_{\frac{a+b}{2}}^{b}\left(\int_{t}^{b} p(s) d s\right) d t & \leq \int_{\frac{a+b}{2}}^{b}\left(\int_{t}^{b} p(s) d s\right) f^{\prime}(t) d t \\
& \leq f_{-}^{\prime}(b) \int_{\frac{a+b}{2}}^{b}\left(\int_{t}^{b} p(s) d s\right) d t
\end{aligned}
$$

and

$$
\begin{aligned}
-f_{-}^{\prime}\left(\frac{a+b}{2}\right) \int_{a}^{\frac{a+b}{2}}\left(\int_{a}^{t} p(s) d s\right) d t & \leq-\int_{a}^{\frac{a+b}{2}}\left(\int_{a}^{t} p(s) d s\right) f^{\prime}(t) d t \\
& \leq-f_{+}^{\prime}(a) \int_{a}^{\frac{a+b}{2}}\left(\int_{a}^{t} p(s) d s\right) d t .
\end{aligned}
$$

If we add these inequalities, then we get

$$
\begin{aligned}
& f_{+}^{\prime}\left(\frac{a+b}{2}\right) \int_{\frac{a+b}{2}}^{b}\left(\int_{t}^{b} p(s) d s\right) d t-f_{-}^{\prime}\left(\frac{a+b}{2}\right) \int_{a}^{\frac{a+b}{2}}\left(\int_{a}^{t} p(s) d s\right) d t \\
& \leq \int_{\frac{a+b}{2}}^{b}\left(\int_{t}^{b} p(s) d s\right) f(t) d t-\int_{a}^{\frac{a+b}{2}}\left(\int_{a}^{t} p(s) d s\right) f(t) d t \\
& \leq f_{-}^{\prime}(b) \int_{\frac{a+b}{2}}^{b}\left(\int_{t}^{b} p(s) d s\right) d t-f_{+}^{\prime}(a) \int_{a}^{\frac{a+b}{2}}\left(\int_{a}^{t} p(s) d s\right) d t
\end{aligned}
$$

Integrating by parts in the Lebesgue integral, we have

$$
\begin{aligned}
\int_{\frac{a+b}{2}}^{b}\left(\int_{t}^{b} p(s) d s\right) d t & \left.=\left(\int_{t}^{b} p(s) d s\right) t\right]_{\frac{a+b}{2}}^{b}+\int_{\frac{a+b}{2}}^{b} t p(t) d t \\
& =\int_{\frac{a+b}{2}}^{b} t p(t) d t-\frac{a+b}{2} \int_{\frac{a+b}{2}}^{b} p(s) d s \\
& =\int_{\frac{a+b}{2}}^{b}\left(t-\frac{a+b}{2}\right) p(t) d t=\frac{1}{2} \int_{a}^{b}\left|t-\frac{a+b}{2}\right| p(t) d t
\end{aligned}
$$

where for the last equality we used the symmetry of $p$. 
Similarly,

$$
\begin{aligned}
\int_{a}^{\frac{a+b}{2}}\left(\int_{a}^{t} p(s) d s\right) d t & \left.=\left(\int_{a}^{t} p(s) d s\right) t\right]_{a}^{\frac{a+b}{2}}-\int_{a}^{\frac{a+b}{2}} p(t) t d t \\
& =\frac{a+b}{2} \int_{a}^{\frac{a+b}{2}} p(s) d s-\int_{a}^{\frac{a+b}{2}} p(t) t d t \\
& =\int_{a}^{\frac{a+b}{2}}\left(\frac{a+b}{2}-t\right) p(t) d t=\frac{1}{2} \int_{a}^{b}\left|t-\frac{a+b}{2}\right| p(t) d t
\end{aligned}
$$

Then by (2.3) we obtain the desired result (2.1).

Remark 1. If we take $p \equiv 1$ in (2.1) and since $\int_{a}^{b}\left|t-\frac{a+b}{2}\right|=\frac{1}{4}(b-a)^{2}$, hence by (2.1) we recapture the inequalities (1.2) from Introduction.

We also have the following refinement and reverse of Fejer's second inequality:

Theorem 3. Let $f$ be a convex function on $I$ and $a, b \in I$, with $a<b$. If $p$ : $[a, b] \rightarrow[a, \infty)$ is Lebesgue integrable and symmetric, namely $p(b+a-t)=p(t)$ for all $t \in[a, b]$, then

$$
\begin{aligned}
0 & \leq \frac{1}{2} \int_{a}^{b}\left[\frac{1}{2}(b-a)-\left|t-\frac{a+b}{2}\right|\right] p(t) d t\left[f_{+}^{\prime}\left(\frac{a+b}{2}\right)-f_{-}^{\prime}\left(\frac{a+b}{2}\right)\right] \\
& \leq\left(\int_{a}^{b} p(t) d t\right) \frac{f(a)+f(b)}{2}-\int_{a}^{b} p(t) f(t) d t \\
& \leq \frac{1}{2} \int_{a}^{b}\left[\frac{1}{2}(b-a)-\left|t-\frac{a+b}{2}\right|\right] p(t) d t\left[f_{-}^{\prime}(b)-f_{+}^{\prime}(a)\right]
\end{aligned}
$$

Proof. Using the integration by parts for Lebesgue integral, we have

$$
\begin{aligned}
& \int_{a}^{b}\left(\int_{a}^{t} p(s) d s-\frac{1}{2} \int_{a}^{b} p(s) d s\right) f^{\prime}(t) d t \\
& \left.=\left(\int_{a}^{t} p(s) d s-\frac{1}{2} \int_{a}^{b} p(s) d s\right) f(t)\right]_{a}^{b}-\int_{a}^{b} p(t) f(t) d t \\
& =\left(\int_{a}^{b} p(s) d s-\frac{1}{2} \int_{a}^{b} p(s) d s\right) f(b)+\left(\frac{1}{2} \int_{a}^{b} p(s) d s\right) f(a) \\
& -\int_{a}^{b} p(t) f(t) d t \\
& =\left(\int_{a}^{b} p(t) d t\right) \frac{f(a)+f(b)}{2}-\int_{a}^{b} p(t) f(t) d t
\end{aligned}
$$


We also have

$$
\begin{aligned}
& \int_{a}^{b}\left(\int_{a}^{t} p(s) d s-\frac{1}{2} \int_{a}^{b} p(s) d s\right) f^{\prime}(t) d t \\
& =\int_{a}^{b}\left(\int_{a}^{t} p(s) d s-\int_{a}^{\frac{a+b}{2}} p(s) d s\right) f(t) d t \\
& =\int_{a}^{\frac{a+b}{2}}\left(\int_{a}^{t} p(s) d s-\int_{a}^{\frac{a+b}{2}} p(s) d s\right) f(t) d t \\
& +\int_{\frac{a+b}{2}}^{b}\left(\int_{a}^{t} p(s) d s-\int_{a}^{\frac{a+b}{2}} p(s) d s\right) f(t) d t \\
& =\int_{\frac{a+b}{2}}^{b}\left(\int_{a}^{t} p(s) d s-\int_{a}^{\frac{a+b}{2}} p(s) d s\right) f(t) d t \\
& -\int_{a}^{\frac{a+b}{2}}\left(\int_{a}^{\frac{a+b}{2}} p(s) d s-\int_{a}^{t} p(s) d s\right) f(t) d t .
\end{aligned}
$$

Observe that

$$
\int_{a}^{t} p(s) d s-\int_{a}^{\frac{a+b}{2}} p(s) d s \geq 0 \text { for } t \in\left[\frac{a+b}{2}, b\right]
$$

and

$$
\int_{a}^{\frac{a+b}{2}} p(s) d s-\int_{a}^{t} p(s) d s \geq 0 \text { for } t \in\left[a, \frac{a+b}{2}\right] .
$$

By the monotonicity of the derivative we have

$$
\begin{aligned}
& f_{+}^{\prime}\left(\frac{a+b}{2}\right) \int_{\frac{a+b}{2}}^{b}\left(\int_{a}^{t} p(s) d s-\int_{a}^{\frac{a+b}{2}} p(s) d s\right) d t \\
& \leq \int_{\frac{a+b}{2}}^{b}\left(\int_{a}^{t} p(s) d s-\int_{a}^{\frac{a+b}{2}} p(s) d s\right) f^{\prime}(t) d t \\
& \leq f_{-}^{\prime}(b) \int_{\frac{a+b}{2}}^{b}\left(\int_{a}^{t} p(s) d s-\int_{a}^{\frac{a+b}{2}} p(s) d s\right) d t
\end{aligned}
$$

and

$$
\begin{aligned}
& -f_{-}^{\prime}\left(\frac{a+b}{2}\right) \int_{a}^{\frac{a+b}{2}}\left(\int_{a}^{\frac{a+b}{2}} p(s) d s-\int_{a}^{t} p(s) d s\right) d t \\
& \leq-\int_{a}^{\frac{a+b}{2}}\left(\int_{a}^{\frac{a+b}{2}} p(s) d s-\int_{a}^{t} p(s) d s\right) f^{\prime}(t) d t \\
& \leq-f_{+}^{\prime}(a) \int_{a}^{\frac{a+b}{2}}\left(\int_{a}^{\frac{a+b}{2}} p(s) d s-\int_{a}^{t} p(s) d s\right) d t .
\end{aligned}
$$


Preprints (www.preprints.org) | NOT PEER-REVIEWED | Posted: 11 September 2019

doi:10.20944/preprints201909.0116.v1

8

S. S. DRAGOMIR

If we add these inequalities, then we get

$$
\begin{aligned}
& {\left[f_{+}^{\prime}\left(\frac{a+b}{2}\right) \int_{\frac{a+b}{2}}^{b}\left(\int_{a}^{t} p(s) d s-\int_{a}^{\frac{a+b}{2}} p(s) d s\right) d t\right.} \\
& \left.-f_{-}^{\prime}\left(\frac{a+b}{2}\right) \int_{a}^{\frac{a+b}{2}}\left(\int_{a}^{\frac{a+b}{2}} p(s) d s-\int_{a}^{t} p(s) d s\right) d t\right] \\
& \leq \int_{\frac{a+b}{2}}^{b}\left(\int_{a}^{t} p(s) d s-\int_{a}^{\frac{a+b}{2}} p(s) d s\right) f^{\prime}(t) d t \\
& -\int_{\frac{a+b}{2}}^{b}\left(\int_{a}^{t} p(s) d s-\int_{a}^{\frac{a+b}{2}} p(s) d s\right) f^{\prime}(t) d t \\
& \leq f_{-}^{\prime}(b) \int_{\frac{a+b}{2}}^{b}\left(\int_{a}^{t} p(s) d s-\int_{a}^{\frac{a+b}{2}} p(s) d s\right) d t \\
& -f_{+}^{\prime}(a) \int_{a}^{\frac{a+b}{2}}\left(\int_{a}^{\frac{a+b}{2}} p(s) d s-\int_{a}^{t} p(s) d s\right) d t .
\end{aligned}
$$

Observe that

$$
\begin{aligned}
& \int_{\frac{a+b}{2}}^{b}\left(\int_{a}^{t} p(s) d s-\int_{a}^{\frac{a+b}{2}} p(s) d s\right) d t \\
& =\int_{\frac{a+b}{2}}^{b}\left(\int_{a}^{t} p(s) d s\right) d t-\frac{b-a}{2} \int_{a}^{\frac{a+b}{2}} p(s) d s \\
& \left.=\left(\int_{a}^{t} p(s) d s\right) t\right]_{\frac{a+b}{2}}^{b}-\int_{\frac{a+b}{2}}^{b} t p(t) d t-\frac{b-a}{2} \int_{a}^{\frac{a+b}{2}} p(s) d s \\
& =b \int_{a}^{b} p(s) d s-\frac{a+b}{2} \int_{a}^{\frac{a+b}{2}} p(s) d s-\int_{\frac{a+b}{2}}^{b} t p(t) d t-\frac{b-a}{2} \int_{a}^{\frac{a+b}{2}} p(s) d s \\
& =b \int_{a}^{b} p(s) d s-b \int_{a}^{\frac{a+b}{2}} p(s) d s-\int_{\frac{a+b}{2}}^{b} t p(t) d t \\
& =b \int_{\frac{a+b}{2}}^{b} p(s) d s-\int_{\frac{a+b}{2}}^{b} t p(t) d t=\int_{\frac{a+b}{2}}^{b}(b-t) p(t) d t
\end{aligned}
$$

and

$$
\begin{aligned}
& \int_{a}^{\frac{a+b}{2}}\left(\int_{a}^{\frac{a+b}{2}} p(s) d s-\int_{a}^{t} p(s) d s\right) d t \\
& =\frac{b-a}{2} \int_{a}^{\frac{a+b}{2}} p(s) d s-\int_{a}^{\frac{a+b}{2}}\left(\int_{a}^{t} p(s) d s\right) d t \\
& \left.=\frac{b-a}{2} \int_{a}^{\frac{a+b}{2}} p(s) d s-\left(\left(\int_{a}^{t} p(s) d s\right) t\right]_{a}^{\frac{a+b}{2}}-\int_{a}^{\frac{a+b}{2}} t p(t) d t\right)
\end{aligned}
$$




$$
\begin{aligned}
& =\frac{b-a}{2} \int_{a}^{\frac{a+b}{2}} p(s) d s-\frac{a+b}{2} \int_{a}^{\frac{a+b}{2}} p(s) d s+\int_{a}^{\frac{a+b}{2}} t p(t) d t \\
& =\int_{a}^{\frac{a+b}{2}} t p(t) d t-a \int_{a}^{\frac{a+b}{2}} p(s) d s=\int_{a}^{\frac{a+b}{2}}(t-a) p(t) d t .
\end{aligned}
$$

If we change the variable $s=b+a-t$, then

$$
\int_{a}^{\frac{a+b}{2}}(t-a) p(t) d t=\int_{\frac{a+b}{2}}^{b}(b-s) p(b+a-s) d s=\int_{\frac{a+b}{2}}^{b}(b-s) p(s) d s .
$$

Finally, observe that

$$
\begin{aligned}
& \frac{1}{2} \int_{a}^{b}\left[\frac{1}{2}(b-a)-\left|t-\frac{a+b}{2}\right|\right] p(t) d t \\
& =\frac{1}{2} \int_{a}^{\frac{a+b}{2}}\left[\frac{1}{2}(b-a)-\left|t-\frac{a+b}{2}\right|\right] p(t) d t \\
& +\frac{1}{2} \int_{\frac{a+b}{2}}^{b}\left[\frac{1}{2}(b-a)-\left|t-\frac{a+b}{2}\right|\right] p(t) d t \\
& =\frac{1}{2} \int_{a}^{\frac{a+b}{2}}\left[\frac{1}{2}(b-a)-\frac{a+b}{2}+t\right] p(t) d t \\
& +\frac{1}{2} \int_{\frac{a+b}{2}}^{b}\left[\frac{1}{2}(b-a)-t+\frac{a+b}{2}\right] p(t) d t \\
& =\frac{1}{2} \int_{a}^{\frac{a+b}{2}}(t-a) p(t) d t+\frac{1}{2} \int_{\frac{a+b}{2}}^{b}(b-t) p(t) d t \\
& =\frac{1}{2} \int_{a}^{\frac{a+b}{2}}(t-a) p(t) d t+\frac{1}{2} \int_{a}^{\frac{a+b}{2}}(t-a) p(t) d t=\int_{a}^{\frac{a+b}{2}}(t-a) p(t) d t
\end{aligned}
$$

and by (2.5) we get (2.4).

Remark 2. Observe that for $p \equiv 1$ we recapture the inequalities (1.3) from Introduction.

If we consider the symmetric weight $p(t)=\left|t-\frac{a+b}{2}\right|, t \in[a, b]$ we obtain from Theorem 2 that

$$
\begin{aligned}
0 & \leq \frac{1}{24}(b-a)^{3}\left[f_{+}^{\prime}\left(\frac{a+b}{2}\right)-f_{-}^{\prime}\left(\frac{a+b}{2}\right)\right] \\
& \leq \int_{a}^{b}\left|t-\frac{a+b}{2}\right| f(t) d t-\frac{1}{4}(b-a)^{2} f\left(\frac{a+b}{2}\right) \\
& \leq \frac{1}{24}(b-a)^{3}\left[f_{-}^{\prime}(b)-f_{+}^{\prime}(a)\right]
\end{aligned}
$$


and from Theorem 3 that

$$
\begin{aligned}
0 & \leq \frac{1}{24}(b-a)^{3}\left[f_{+}^{\prime}\left(\frac{a+b}{2}\right)-f_{-}^{\prime}\left(\frac{a+b}{2}\right)\right] \\
& \leq(b-a)^{2} \frac{f(a)+f(b)}{8}-\int_{a}^{b}\left|t-\frac{a+b}{2}\right| f(t) d t \\
& \leq \frac{1}{24}(b-a)^{3}\left[f_{-}^{\prime}(b)-f_{+}^{\prime}(a)\right],
\end{aligned}
$$

where $f$ is convex on $[a, b]$. These provide refinements and reverses of the inequalities (1.5).

If we consider the symmetric weight $p(t)=(t-a)(b-t), t \in[a, b]$ we obtain from Theorem 2 that

$$
\begin{aligned}
0 & \leq \frac{7}{192}(b-a)^{4}\left[f_{+}^{\prime}\left(\frac{a+b}{2}\right)-f_{-}^{\prime}\left(\frac{a+b}{2}\right)\right] \\
& \leq \int_{a}^{b}(t-a)(b-t) f(t) d t-\frac{1}{6}(b-a)^{3} f\left(\frac{a+b}{2}\right) \\
& \leq \frac{7}{192}(b-a)^{4}\left[f_{-}^{\prime}(b)-f_{+}^{\prime}(a)\right]
\end{aligned}
$$

and from Theorem 3 that

$$
\begin{aligned}
0 & \leq \frac{7}{192}(b-a)^{4}\left[f_{+}^{\prime}\left(\frac{a+b}{2}\right)-f_{-}^{\prime}\left(\frac{a+b}{2}\right)\right] \\
& \leq(b-a)^{3} \frac{f(a)+f(b)}{12}-\int_{a}^{b}(t-a)(b-t) f(t) d t \\
& \leq \frac{7}{192}(b-a)^{4}\left[f_{-}^{\prime}(b)-f_{+}^{\prime}(a)\right],
\end{aligned}
$$

where $f$ is convex on $[a, b]$. These provide refinements and reverses of the inequalities (1.6).

\section{REFERENCES}

[1] E. F. Beckenbach, Convex functions, Bull. Amer. Math. Soc. 54 (1948), 439-460.

$[2]$ S. S. Dragomir, An inequality improving the first Hermite-Hadamard inequality for convex functions defined on linear spaces and applications for semi-inner products, J. Inequal. Pure Appl. Math. 3 (2002), No. 2, Article 31. [Online https://www.emis.de/journals/JIPAM/article183.html?sid=183] .

[3] S. S. Dragomir, An inequality improving the second Hermite-Hadamard inequality for convex functions defined on linear spaces and applications for semi-inner products, J. Inequal. Pure Appl. Math. 3 (2002), No. 3, Article 35. [Online https://www .emis.de/journals/JIPAM/article187.html?sid=187] .

[4] S. S. Dragomir, Ostrowski type inequalities for Lebesgue integral: a survey of recent results. Aust. J. Math. Anal. Appl. 14 (2017), no. 1, Art. 1, 283 pp. [Online http://ajmaa.org/cgi-bin/paper.pl?string=v14n1/V14I1P1.tex] .

[5] S. S. Dragomir and C. E. M. Pearce, Selected Topics on HermiteHadamard Inequalities and Applications, RGMIA Monographs, 2000. [Online http://rgmia.org/monographs/hermite_hadamard.html] .

[6] L. Féjer, Über die Fourierreihen, II, (In Hungarian). Math. Naturwiss, Anz. Ungar. Akad. Wiss., 24 (1906), 369-390.

[7] D. S. Mitrinović and I. B. Lacković, Hermite and convexity, Aequationes Math. 28 (1985), 229-232.

[8] A. W. Roberts and D. E. Varberg, Convex Functions, Academic Press, 1973. 
${ }^{1}$ Mathematics, College of Engineering \& Science, Victoria University, PO Box 14428 , Melbourne City, MC 8001, Australia.

E-mail address: sever.dragomir@vu.edu.au

URL: http://rgmia.org/dragomir

2DSt-nRF Centre of Excellence in the Mathematical, and Statistical Sciences, School of Computer Science, \& Applied Mathematics, University of the Witwatersrand,, Johanesburg, South Africa. 